\title{
Anti-cancer effects of a novel Pan-RAF inhibitor in a hepatocellular carcinoma cell line
}

\author{
WEI WANG ${ }^{1}$, BO XU ${ }^{2}$, QIXIANG $\mathrm{LI}^{3}$, DECHUN JIANG ${ }^{1}$ and SUYING YAN ${ }^{1}$ \\ ${ }^{1}$ Department of Pharmacy, Xuanwu Hospital, Capital Medical University, Beijing 100053; \\ ${ }^{2}$ State Key Laboratory of Natural and Biomimetic Drugs, Peking University Health Science Center, Beijing 100191; \\ ${ }^{3}$ Biomarker and Diagnostic Technology, Crown Bioscience Inc., Beijing 102200, P.R. China
}

Received March 16, 2017; Accepted February 1, 2018

DOI: $10.3892 / \mathrm{mmr} .2018 .8615$

\begin{abstract}
The RAF/mitogen-activated protein kinase (MAPK)/extracellular signal-regulated kinase (ERK) kinase (MEK)/ERK (RAF/MEK/ERK) signaling cascade serves a prominent role in hepatocellular carcinoma (HCC) proliferation. Sorafenib (BAY 43-9006) is a potent multikinase inhibitor of RAF kinases and a few receptor tyrosine kinases. Additionally, sorafenib causes apoptosis in a number of human tumor cell lines such as leukemia cell lines. Sorafenib is the first targeted drug to prolong the overall survival of patients with advanced HCC. However, sorafenib activity is less favorable in certain cancers, including sarcomas and melanomas, due to patient insensitivity and drug resistance. In the present study, a novel bi-aryl urea, N-(3-trifluoromethylphenyl)-N'-(2-methyl4-(6-cyclopropanecarboxamido-pyrimidin-4-yl) oxyphenyl) urea (CBI-5725), is shown to be a potential candidate for the treatment of liver cancer. In the present study, the in vitro activities of CBI-5725 and sorafenib in PLC/PRF/5 HCC cells were examined and the corresponding in vivo antitumor activities in PLC/PRF/5 human tumor xenografts. An alamar blue assay confirmed that CBI-5725 was more cytotoxic than sorafenib to PLC/PRF/5 cells, suggesting that CBI-5725 inhibited tumor cell proliferation more potently than sorafenib. CBI-5725 inhibited the RAF/MEK/ERK signaling pathway to the same extent as sorafenib. In addition, CBI-5725 elicited cell cycle arrest in the G2/M phase, while sorafenib did not markedly alter the cell cycle. Furthermore, CBI-5725 induced apoptosis more strongly than sorafenib in a dose-dependent manner, which may be attributed to greater caspase-3 and poly(adenosine 5'-diphosphate-ribose) polymerase activation by CBI-5725. In the PLC/PRF/5 xenograft model, $2 \mathrm{mg} / \mathrm{kg}$
\end{abstract}

Correspondence to: Professor Suying Yan, Department of Pharmacy, Xuanwu Hospital, Capital Medical University, 45 Changchun Street, Xicheng, Beijing 100053, P.R. China E-mail: yansuying10@sina.cn

Key words: hepatocellular carcinoma, CBI-5725, sorafenib, Raf kinase/mitogen-activated protein kinase kinase/extracellular signal-regulated kinase, apoptosis, cell cycle
CBI-5725 inhibited tumor growth by $73 \%$. At doses ranging from 6 to $18 \mathrm{mg} / \mathrm{kg}$, CBI-5725 nearly completely prevented tumor growth. These results imply that the antitumor efficacy of CBI-5725 in HCC models may result from the suppression of the RAF/MEK/ERK signaling pathway, the induction of cell cycle arrest in the G2/M phase, and the initiation of caspase-3-dependent apoptosis. These observations suggested that CBI-5725 may be a potent novel compound for the treatment of HCC.

\section{Introduction}

Hepatocellular carcinoma (HCC) is recognized as the sixth most common cancer in the world and the third leading cause of cancer-related deaths (1). HCC occurs at a higher annual incidence in Asia and Africa than in western countries, and finding effective treatments has therefore been a top priority in these countries. Since HCC is caused by chronic liver disease and is often diagnosed at an advanced stage, drugs are one of the main treatment options for patients with advanced HCC. Chemotherapeutics such as doxorubicin are currently the primary agents used to treat HCC; however, these cytotoxic molecules are non-selective and cause severe side effects (2). Therefore, researchers have begun to investigate targeted therapeutics. Sorafenib is the only approved targeted drug for HCC patients (2). It inhibits RAF serine/threonine kinases (mutant and wild-type B-RAF and C-RAF/Raf-1), which play a pivotal role in mitogenic and oncogenic signal transduction through the RAF/mitogen-activated protein kinase (MAPK)/extracellular signal-regulated kinase (ERK) kinase (MEK)/ERK ( $\mathrm{RAF} / \mathrm{MEK} / \mathrm{ERK}$ ) cascade involved in tumor proliferation (3). In addition, sorafenib strongly inhibits tyrosine kinase receptors that promote angiogenesis, such as vascular endothelial growth factor receptor (VEGFR)2, VEGFR 3, platelet-derived growth factor receptor- $\beta$ (PDGFR- $\beta$ ), Flt3, and c-Kit (4). In addition to blocking the RAF/MEK/ERK pathway, sorafenib induces tumor cell apoptosis in hepatocellular PLC/PRF/5 cells independent of caspase activation (3). Though sorafenib is the only available therapeutic agent that can prolong the overall survival of patients with advanced HCC, sorafenib resistance in HCC often prevents its long-term efficacy (5). Generally, resistance to sorafenib can be attributed to two causes. First, HCC cells can harbor intrinsic resistance prior to drug treatment. 
Ezzoukhry et al (6) reported that the sensitivity of different types of HCC cells to sorafenib is diverse and suggested that the drug resistance of some HCC cell lines (e.g., Hep3B) may occur spontaneously when sorafenib is used at clinically relevant concentrations (6). Additionally, previous studies have demonstrated the paradoxical ability of sorafenib to activate RAF kinases by transactivating RAF dimers (7). The biochemical rationale for sorafenib-induced activation of RAF kinases seems to be in line with the mechanisms underlying intrinsic sorafenib resistance in HCC. Second, acquired resistance to sorafenib during the course of therapy is often encountered in HCC (5,8-10). For example, somatic mutations in oncogenes and the activation of numerous oncogenic signaling pathways, e.g., the MAPK/RAS/ERK, TGF $\beta$, and PI3K/PTEN/AKT pathways, contribute to the development of sorafenib resistance (2). With the emergence of sorafenib, clinical studies have been performed with a range of drugs, primarily molecular targeted therapies; however, these trials have terminated in failure (11). Despite this difficulty, there is still an urgent need to identify novel pharmaceutical drugs beyond sorafenib. CBI-5725 is a novel bi-aryl urea; the patent for this compound is owned by Crown Bioscience, Inc. (China). Due to its potent inhibitory effect on HCC cell proliferation (Fig. 1), CBI-5725 was selected for further investigation. In this paper, the anti-cancer effects of this compound on HCC tumor cells were assessed in vitro and compared with those of sorafenib in the PLC/PRF/5 (mutant K-RAS and wild-type B-RAF) HCC cell line. Furthermore, the mechanism underlying CBI-5725 activity was explored. A comparison of the anti-cancer effects of CBI-5725 and sorafenib is of great importance for understanding the underlying mechanism. The aim of this investigation was to identify a novel multikinase inhibitor as a potential candidate for the treatment of liver cancer.

\section{Materials and methods}

Compounds. Sorafenib was purchased from Cell Signaling Technology, Inc. (Danvers, MA, USA). CBI-5725 was synthesized by Crown Bioscience, Inc. (San Diego, CA, USA). The chemical name of CBI-5725 is N-(3-trifluoromethylphenyl)N'-(2-methyl-4-(6-cyclopropanecarboxamido-pyrimidin-4-yl) oxyphenyl) urea. The structural formula is displayed in Fig. 1. The compounds were dissolved in 100\% DMSO (Applichem, Darmstadt, Germany) and diluted in minimum essential medium (MEM; MP Biomedicals, Solon, OH, USA) to a range of concentrations with a final DMSO concentration of $0.1 \%$. Cells were treated with $0.1 \%$ (v/v) DMSO as a solvent control.

Cell lines. PLC/PRF/5 (mutant p53, mutant K-RAS, and wild-type B-RAF) human HCC cells were purchased from American Type Culture Collection (Manassas, VA, USA) and incubated in MEM with $10 \%$ fetal bovine serum (FBS; PAA, Austria) in a $5 \% \mathrm{CO}_{2}$ atmosphere at $37^{\circ} \mathrm{C}$.

Alamar blue assay. Tumor cells were trypsinized, plated at a density of 5,000 cells per well in 96-well plates and cultured overnight in a humidified chamber with $5 \% \mathrm{CO}_{2}$ at $37^{\circ} \mathrm{C}$. The next day, compounds were added to the wells at a final concentration ranging from $7.6 \mathrm{nM}$ to $50 \mu \mathrm{M}$. The cells were treated with test compounds for $72 \mathrm{~h}$ at $37^{\circ} \mathrm{C}$. Subsequently, alamar blue was added at a 10 -fold dilution in complete growth medium. This assay evaluates the number of viable cells per well based on fluorescent signal measurements.

Cellular B-RAF, C-RAF, MEK1/2, ERK1/2, caspase-3, poly(ADP-ribose) polymerase (PARP), and Akt activation. Cells were plated at a density of 600,000 cells per dish in $60 \times 15 \mathrm{~mm}$ tissue culture dishes (Nunclon; Thermo Fisher Scientific, Inc., Waltham, MA, USA). The following day, cells were washed once with serum-free medium and treated with compounds in MEM with 10\% FBS for $2 \mathrm{~h}$. Afterwards, cells were washed with cold PBS containing $0.1 \mathrm{mM}$ vanadate (Santa Cruz Biotechnology, Inc., Dallas, TX, USA) to inhibit dephosphorylation and then lysed in RIPA lysis buffer (Applygen Technologies, Inc., Beijing, China) containing protease inhibitor cocktail tablets (Roche Diagnostics, Basel, Switzerland). The lysates were centrifuged, and $90 \mu \mathrm{g}$ of soluble protein was separated by SDS-PAGE, transferred to PVDF membranes (Hybond-C extra; Amersham Pharmacia Biotech, Piscataway, NJ, USA) and blocked in TBS-BSA (Amresco LLC, Solon, $\mathrm{OH}$, USA) (for phosphorylated proteins) or TBS-milk. The membranes were probed with anti-pB-RAF, anti-pC-RAF, anti-pMEK1/2, anti-pERK1/2, anti-caspase-3, and anti-PARP primary antibodies (Cell Signaling Technology, Inc.) and horseradish peroxidase (HRP)-conjugated secondary antibodies (Santa Cruz Biotechnology, Inc.). The membranes were then treated with an enhanced chemiluminescence reagent (EMD Millipore, Billerica, MA, USA), and the protein signals were detected by an EC3 imaging system (UVP EC3 Imaging System, UVP Inc., Upland, CA, USA).

Cell cycle analysis. Flow cytometry was utilized to determine the cell cycle distribution. Cells $\left(5 \times 10^{5}\right)$ were plated into 6 -well plates, incubated with test compounds for $24 \mathrm{~h}$, collected, fixed in cold $70 \%$ ethanol overnight at $-20^{\circ} \mathrm{C}$, washed once with PBS, and finally stained with propidium iodide (PI) solution (50 $\mu \mathrm{g} / \mathrm{ml} \mathrm{PI} \mathrm{and} 50 \mu \mathrm{g} / \mathrm{ml}$ RNase A in PBS) for $30 \mathrm{~min}$ in the dark. The samples were then examined by flow cytometry.

Annexin V-fluorescein isothiocyanate (FITC)/PI apoptosis assay. FITC-conjugated Annexin V (Annexin V-FITC)/PI double staining was used to quantitatively examine the proportion of apoptotic cells. Annexin V detects externalized phosphatidylserine, a marker of early apoptosis, while PI binds to nuclear DNA and is indicative of the loss of plasma membrane integrity associated with late apoptosis $(12,13)$. Early apoptotic cells are Annexin V positive and PI negative (Annexin $\mathrm{V}$-FITC ${ }^{+} / \mathrm{PI}^{-}$), whereas late apoptotic cells are Annexin V/PI double-positive (Annexin V-FITC ${ }^{+} / \mathrm{PI}^{+}$) $(14,15)$. The total number of apoptotic cells is the sum of early and late apoptotic cells (16-18). Cells were treated with tested compounds for $48 \mathrm{~h}$, harvested, washed twice with PBS and then resuspended in $500 \mu \mathrm{l}$ of binding buffer. A total of $5 \mu \mathrm{l}$ of Annexin V-FITC was added to the cells, which were then incubated at room temperature for $10 \mathrm{~min}$. Subsequently, $5 \mu 1$ of PI was added, and the cells were incubated in the dark for another $10 \mathrm{~min}$ before analysis by flow cytometry.

Tumor xenograft experiments. Male NCr-nu/nu mice, aged 5 weeks, were purchased from Vital River (Beijing, China), 
for the in vivo study. The mice were housed and received water and food ad libitum. Experiments using these mice were performed in accordance with protocols approved by the Animal Ethical Committee of Xuanwu Hospital. Tumors were generated by harvesting PLC/PRF/5 cells from mid-log phase cultures using trypsin-EDTA (Invitrogen; Thermo Fisher Scientific, Inc.). PLC/PRF/5 cells $\left(5 \times 10^{6}\right)$ suspended in $50 \%$ Matrigel (BD Biosciences, Franklin Lakes, NJ, USA) in serum-free medium were injected s.c. into the dorsal flank of each mouse. Sorafenib tosylate and CBI-5725 were dissolved in Cremophor EL/95\% ethanol (50:50; Sigma-Aldrich; Merck KGaA, Darmstadt, Germany). When PLC/PRF/5 tumors reached 200 to $220 \mathrm{~mm}^{3}$, sorafenib tosylate was administered p.o. once daily for 14 days at 8 or $20 \mathrm{mg} / \mathrm{kg}$ body weight. CBI-5725 was administered p.o. once daily for 14 days at 2, 6, or $15 \mathrm{mg} / \mathrm{kg}$ body weight. The control group received vehicle treatment. Tumors were measured using a Vernier caliper, and tumor volume was determined by measuring the longest (a) and shortest (b) diameters and was calculated using the equation $\mathrm{axb}^{2} \mathrm{x} \pi / 6$.

Statistical analysis. All experiments were conducted at least three independent times. The data were examined by one-way ANOVA, and individual groups were compared by Tukey's post hoc test. Statistical significance was determined at a confidence interval of at least $95 \%$.

\section{Results}

CBI-5725 inhibits PLC/PRF/5 cell proliferation. Alamar blue was used to measure the inhibitory effect of CBI-5725 on PLC/PRF/5 cell growth. Increased cell death and debris, decreased cell density, and cell shrinkage were observed after 72 h of CBI-5725 treatment. As shown in Fig. 1, the inhibitory effect of CBI-5725 on PLC/PRF/5 cell growth occurred in a dose-dependent manner. The $\mathrm{IC}_{50}$ of CBI-5725 in PLC/PRF/5 cells was $0.83 \pm 0.09 \mu \mathrm{M}$, whereas the $\mathrm{IC}_{50}$ of sorafenib was $4.99 \pm 0.13 \mu \mathrm{M}$, indicating that $\mathrm{CBI}-5725$ is more cytotoxic than sorafenib.

CBI-5725 inhibits the RAF/MEK/ERK signaling cascade in $P L C / P R F / 5$ cells. RAF kinases are essential regulators of the MEK/ERK cascade, which regulates multiple physiological functions required for cell growth and survival (19). However, overactivation of this pathway induces abnormal cell growth and malignant behavior in human tumors. Abnormal tumor growth is usually induced by the activation of ERK signaling via mutations in RAS and RAF $(20,21)$. Up-regulated $\mathrm{RAF} / \mathrm{MEK} / \mathrm{ERK}$ signaling plays a prominent role in HCC (22). To compare the effects of CBI-5725 and sorafenib on $\mathrm{RAF} / \mathrm{MEK} / \mathrm{ERK}$ signal transduction in PLC/PRF/5 cells, Western blot analyses were performed to examine alterations in the phosphorylation level of key proteins in this pathway. As shown in Figs. 2, 3A and 3B, neither CBI-5725 nor sorafenib changed the level of phosphorylated B-RAF, but both drugs markedly increased basal C-RAF phosphorylation in a dose-dependent manner. Although both CBI-5725 and sorafenib paradoxically enhanced C-RAF activation, they both suppressed the phosphorylation of MEK and ERK in a dose-dependent manner (Figs. 2, 3C and 3D), suggesting
A



B
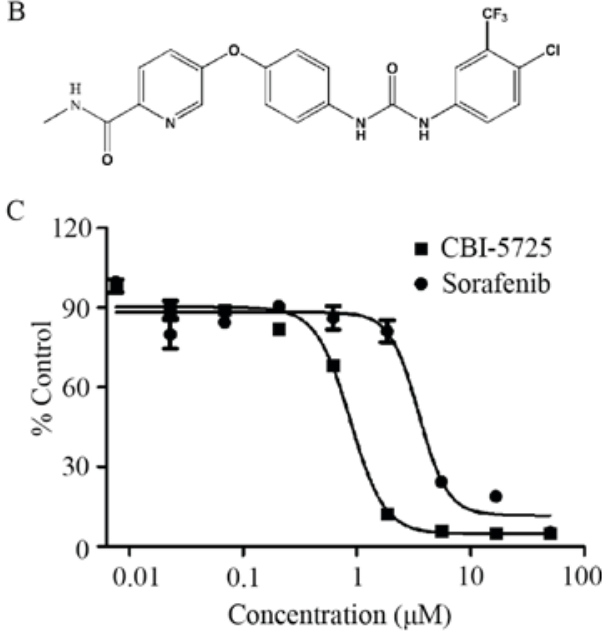

Figure 1. Inhibitory effect of CBI-5725 or sorafenib on PLC/PRF/5 cells (A) Chemical structure of CBI-5725. (B) Chemical structure of sorafenib. (C) Inhibition of cell proliferation. Cells were treated with CBI-5725 or sorafenib and incubated for $72 \mathrm{~h}$. Data are presented as the mean \pm standard error mean of three independent experiments.

that both CBI-5725 and sorafenib are pan-RAF inhibitors. The ratio of each phosphorylated protein to total protein was examined by one-way ANOVA followed by Tukey's post hoc test, and the data indicated that the effects of CBI-5725 and sorafenib on the RAF/MEK/ERK cascade were comparable (Fig. 3A-D). Total B-RAF, C-RAF, MEK, ERK and Akt levels were unaltered, and no changes were detected in phospho-Akt levels (Fig. 3E).

CBI-5725 modifies the cell cycle. In eukaryotic cells, the cell cycle is a highly conserved mechanism by which replication occurs (23). Irreversible cell cycle arrest serves as an alternative mechanism to prevent continued proliferation in cancer (24). As the above studies suggested that CBI-5725 prevents PLC/PRF/5 cell proliferation, the effect of CBI-5725 on cell cycle progression was examined. As shown in Fig. 4, CBI-5725 (15 $\mu \mathrm{M}$; Fig. 4C) significantly increased the proportion of cells in $\mathrm{G} 2 / \mathrm{M}$ phase to $(35.77 \pm 1.08) \%$, whereas only $(24.65 \pm 0.94) \%$ and $(15.92 \pm 1.39) \%$ of cells were in $\mathrm{G} 2 / \mathrm{M}$ phase in the control (Fig. 4A) and sorafenib (15 $\mu \mathrm{M}$; Fig. 4B) groups, respectively. The result in Fig. 4D indicates that cells treated for $24 \mathrm{~h}$ with CBI-5725 were arrested in G2/M phase. In contrast, although sorafenib $(15 \mu \mathrm{M})$ increased the percentage of cells in G0/G1 phase, there was no significant difference in the cell cycle distribution of sorafenib-treated cells and control cells.

CBI-5725 induces the apoptosis of PLC/PRF/5 cells. Because the cell cycle and apoptosis are intimately linked, the effect of CBI-5725 and sorafenib on the apoptosis of PLC/PRF/5 cells was examined using Annexin V-FITC and PI staining and flow cytometry. As shown in Fig. 5, each quadrant in the flow cytometry histogram represents a specific cell population. The lower left quadrant exhibits live cells (negative 




Figure 2. Effect of CBI-5725 or sorafenib on RAF/MEK/ERK signaling in PLC/PRF/5 cells. Cells were incubated with different concentrations of CBI-5725 or sorafenib for $2 \mathrm{~h}$. Protein phosphorylation was examined by western blot analysis. RAF, Raf kinase; MEK, mitogen-activated protein kinase (MAPK)/ERK kinase (ERK); p, phosphorylated; Akt, protein kinase B.
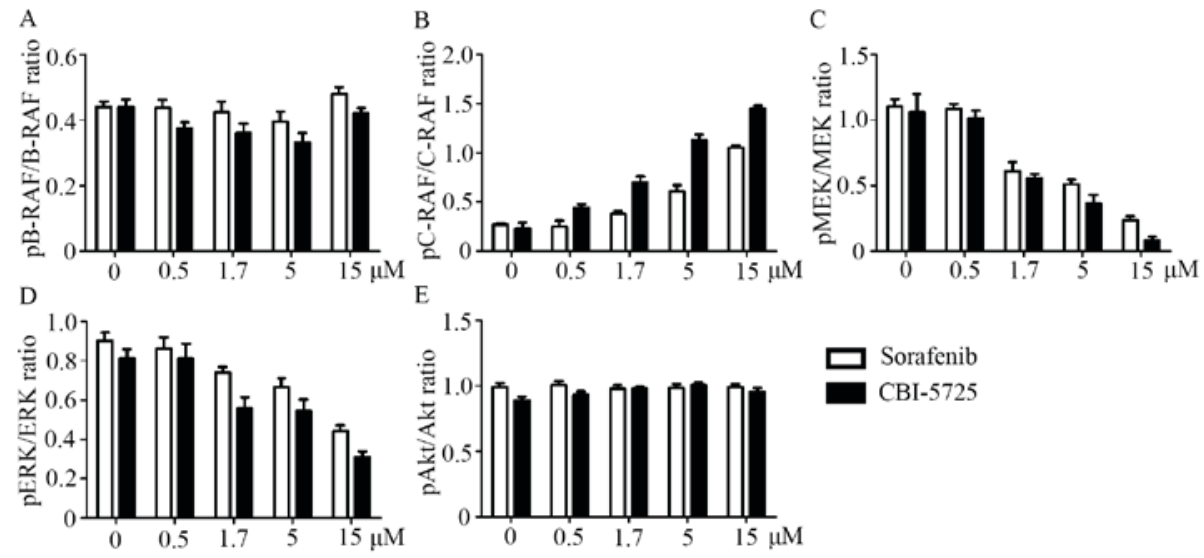

Figure 3. Densitometric analyses of phosphorylated proteins in the RAF/MEK/ERK signal transduction pathway in PLC/PRF/5 cells. The white and black bars represent the densitometric intensity of each phosphorylated protein relative to that of the corresponding total protein. (A) pB-RAF, (B) pC-RAF, (C) pMEK, (D) pERK and (E) pAkt. Data are presented as the mean \pm standard error mean of three independent experiments. RAF, Raf kinase; MEK, mitogen-activated protein kinase (MAPK)/ERK kinase (ERK); p, phosphorylated; Akt, protein kinase B.

for Annexin V and PI), the lower right quadrant shows early apoptotic cells, the upper right quadrant displays late apoptotic cells, and the upper left quadrant exhibits dead cells/debris (positive for PI only). When PLC/PRF/5 cells were treated with CBI-5725 (5 or $15 \mu \mathrm{M})$ for $48 \mathrm{~h}$, the proportion of total apoptotic cells was $28.91 \pm 1.13$ and $46.95 \pm 1.12 \%$, respectively; both percentages were higher than that for control treatment $(5.47 \pm 0.75 \%)$. These findings indicate that CBI-5725 induces the apoptosis of PLC/PRF/5 cells in a dose-dependent manner. Fig. 5E shows that CBI-5725 $(15 \mu \mathrm{M})$ induced more apoptosis than sorafenib $(15 \mu \mathrm{M})$ $(46.95 \pm 1.12$ vs. $25.50 \pm 1.34 \%, \mathrm{P}<0.01)$. 

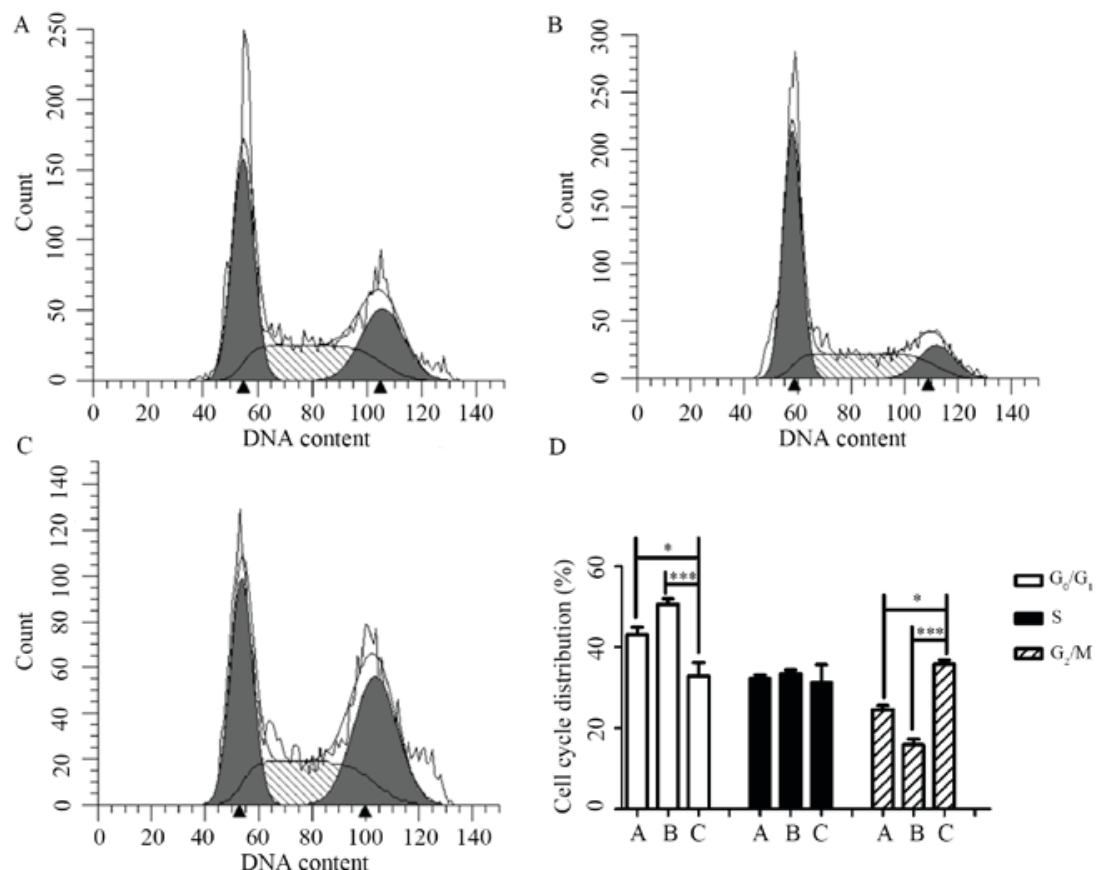

Figure 4. Effect of CBI-5725 or sorafenib on the cell cycle distribution of PLC/PRF/5 cells. Cells were treated with (A) control, (B) $15 \mu \mathrm{M}$ sorafenib or (C) $15 \mu \mathrm{M}$ CBI-5725 for $24 \mathrm{~h}$ and stained with propidium iodide. (D) The cell cycle distribution was evaluated by flow cytometry and analyzed using Modfit software. Bars A-C represent the treatment data presented in parts A-C of the figure. The columns indicate the mean \pm standard error mean of apoptotic cells obtained from three independent experiments. ${ }^{*} \mathrm{P}<0.05$ and ${ }^{* * * *} \mathrm{P}<0.001$, as indicated.

A

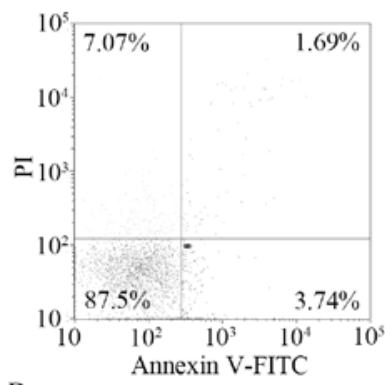

$\mathrm{D}$

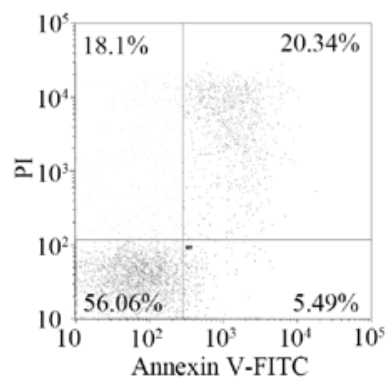

B


$\mathrm{C}$

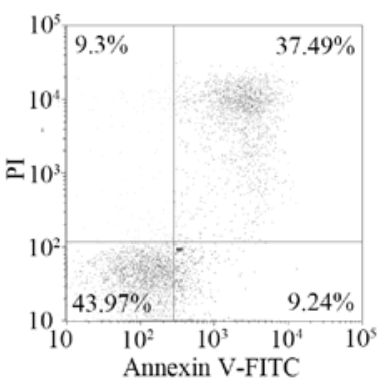

Annexin V-FITC

Figure 5. CBI-5725- or sorafenib-induced apoptosis was detected by Annexin V-FITC/PI staining assay. PLC/PRF/5 cells were incubated with different concentrations of CBI-5725 or sorafenib for $48 \mathrm{~h}$. The cells were then stained with Annexin V and PI, and subsequently analyzed by flow cytometry: (A) Control, (B) $5 \mu \mathrm{M}$ CBI-5725, (C) $15 \mu \mathrm{M}$ CBI-5725 and (D) $15 \mu \mathrm{M}$ sorafenib. (E) The percentage of apoptotic cells treated as described in parts A-D. The sum of early apoptotic cells (Annexin V-FITC $/ \mathrm{PI}^{-}$) and late apoptotic cells (Annexin V-FITC $/ \mathrm{PI}^{+}$) encompassed the total apoptotic population. The data are presented as the mean \pm standard error mean of three independent experiments. ${ }^{* *} \mathrm{P}<0.01$, as indicated. FITC, fluorescein isothiocyanate; PI, propidium iodide.

Apoptosis induced by CBI-5725 in PLC/PRF/5 cells relies on initiation of the caspase-3 signaling pathway. Caspases, as critical effectors of apoptosis, are divided into upstream initiator caspases and downstream executioner caspases (25). There are two common pathways by which caspases can be activated. Caspase- 9 is the upstream caspase for the intrinsic pathway, while caspase- 8 has this role in the extrinsic pathway (26). Both pathways converge on caspase-3, a downstream executioner caspase. Once activated, pro-caspase- 3 is cleaved, giving rise to the active form of caspase-3. Active caspase-3 cleaves PARP, ultimately leading to apoptosis (27). Cleaved PARP is among the most used biomarkers of 


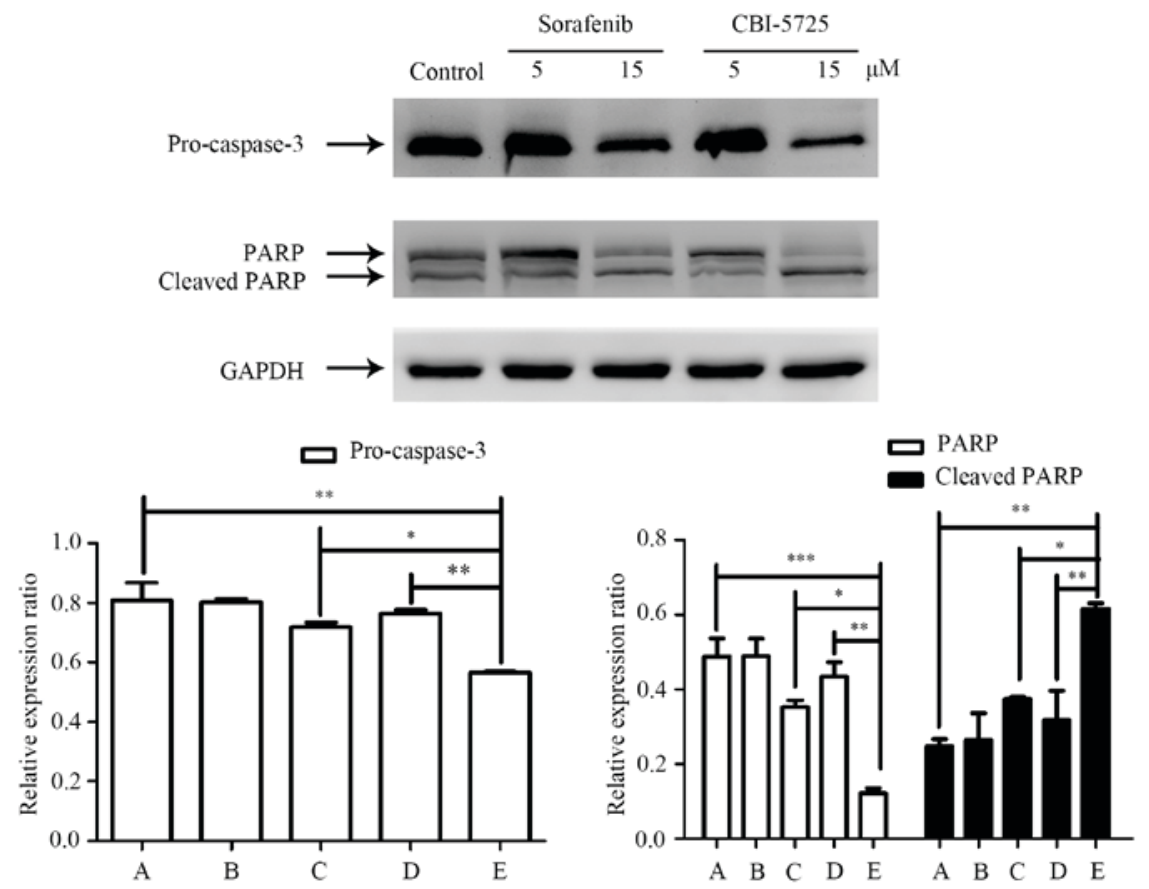

Figure 6. Effect of CBI-5725 or sorafenib on PARP and caspase-3 levels in PLC/PRF/5 cells. PLC/PRF/5 cells were incubated with or without the test compounds for $48 \mathrm{~h}$, collected and lysed. Protein expression was determined by western blot analyses. The bars indicate the densitometric saturation of the corresponding protein bands relative to that of GAPDH (set at an intensity of $100 \%$ ). A, Control; B, $5 \mu \mathrm{M}$ sorafenib; C, $15 \mu \mathrm{M}$ sorafenib; D, $5 \mu \mathrm{M}$ CBI-5725; and $\mathrm{E}, 15 \mu \mathrm{M}$ CBI-5725. The data are presented as the mean \pm standard error mean of three separate experiments. ${ }^{*} \mathrm{P}<0.05,{ }^{* * *} \mathrm{P}<0.01$ and ${ }^{* * * *} \mathrm{P}<0.001$, as indicated. PARP, poly(adenosine 5'-diphosphate ribose) polymerase.


Figure 7. In vivo effect of (A) CBI-5725 or (B) sorafenib on a PLC/PRF/5 xenograft nude mouse model. PLC/PRF/5 tumor cells were implanted subcutaneously into the dorsal flank of each mouse. Treatment was initiated on day 12 when all mice had tumors ranging in size from 200 to $220 \mathrm{~mm}^{3}$. CBI-5725 or sorafenib tosylate was administered orally once daily for 14 days at the indicated dose. There was no lethality in any treatment or control group. Data are presented as the mean \pm standard error mean $(\mathrm{n}=6) .{ }^{* *} \mathrm{P}<0.01$ and ${ }^{* * *} \mathrm{P}<0.001$ vs. vehicle group.

apoptosis (28). Changes in caspase-3 and PARP levels in $\mathrm{PLC} / \mathrm{PRF} / 5$ cells after treatment with CBI-5725 or sorafenib were measured by Western blot analyses. As shown in Fig. 6, a dose-dependent decrease in pro-caspase-3 and PARP levels and an increase in cleaved PARP levels were observed in cells after $48 \mathrm{~h}$ of CBI-5725 treatment. However, pro-caspase-3, PARP and cleaved PARP levels were not distinctly altered in cells treated with sorafenib. As shown in Fig. 6D and E, pro-caspase-3 and PARP levels were much lower in cells treated with $15 \mu \mathrm{M}$ CBI-5725 than in control cells $(0.566 \pm 0.004$ vs. $0.807 \pm 0.061, \mathrm{P}<0.01 ; 0.121 \pm 0.014$ vs. $0.487 \pm 0.05, \mathrm{P}<0.0001$ ), but cleaved PARP levels were much higher in CBI-5725-treated cells than in control cells $(0.615 \pm 0.015$ vs. $0.247 \pm 0.019, \mathrm{P}<0.01)$. However, in cells treated with $15 \mu \mathrm{M}$ sorafenib, there were no significant changes in these levels (pro-caspase-3: $0.718 \pm 0.015$ vs. $0.807 \pm 0.061$; PARP: $0.352 \pm 0.018$ vs. $0.487 \pm 0.05$; cleaved PARP: $0.374 \pm 0.006$ vs. $0.247 \pm 0.019$ ). These data are consistent with the results of the Annexin V-FITC/PI staining assay. The two main apoptotic pathways both involve the activation of caspase- 3 . The results indicate that the CBI-5725-induced apoptosis of PLC/PRF/5 cells occurs via the activation of caspase-3 and PARP.

CBI-5725 inhibits PLC/PRF/5 xenograft tumor growth in vivo. To verify whether the effect of CBI-5725 on PLC/PRF/5 cells has clinical relevance, we treated HCC xenografts with CBI-5725 to assess its in vivo effect in comparison with that of sorafenib. A single tumor was observed in each mouse used in the experiments. As shown in Fig. 7A, CBI-5725 significantly 
inhibited PLC/PRF/5 xenograft tumor growth. In comparison with the vehicle group, in which the largest tumor diameter was $21 \mathrm{~mm}, 2 \mathrm{mg} / \mathrm{kg}$ CBI-5725 inhibited tumor growth by approximately $73 \%$ at the end of treatment (the largest tumor diameter was $13.4 \mathrm{~mm}$ ), and 6 and $18 \mathrm{mg} / \mathrm{kg} \mathrm{CBI}-5725$ caused 89 and $92 \%$ tumor growth inhibition, respectively (the largest tumor diameters were 10.7 and $10.3 \mathrm{~mm}$, respectively). Sorafenib tosylate inhibited PLC/PRF/5 tumor growth in a dose-dependent manner. At 10 and $20 \mathrm{mg} / \mathrm{kg}$, sorafenib inhibited tumor growth by 19 and 64\%, respectively (the largest tumor diameters were 19.5 and $15 \mathrm{~mm}$, respectively). There was no increased weight loss in each treatment group relative to the control group.

\section{Discussion}

Sorafenib is a multikinase inhibitor that acts in opposition to the Ser/Thr kinase RAF and to a number of receptor tyrosine kinases (RTKs), such as VEGFR2 and PDGFR, which are essential for tumor cell proliferation and angiogenesis. Moreover, sorafenib induces the apoptosis of PLC/PRF/5 cells (3). Although sorafenib is the only approved targeted drug for HCC patients, increased sorafenib resistance in HCC reduces its efficacy (29). Since the advent of sorafenib, many trials for novel treatments for advanced HCC, primarily focused on molecular targeted therapy, have been conducted; however, with the exception of regorafenib, the results to date have been disappointing (11). The difficulty in advancing a new drug or treatment method can be attributed to the diverse mechanisms of HCC carcinogenesis and progression, as well as to the existence of background liver diseases, such as chronic hepatitis and cirrhosis. Despite these failures, novel pharmaceutical drugs are still urgently needed. In this study, CBI-5725 was shown to more potently prevent the proliferation and induce the apoptosis of a human HCC cell line compared to sorafenib. CBI-5725 blocked the RAF/MEK/ERK pathway, inhibited the cell cycle at G2/M phase and induced apoptosis dependent on caspase 3/PARP activation. In addition, CBI-5725 exerted robust antitumor activity by inhibiting the growth of PLC/PRF/5 xenografts. Therefore, CBI-5725 is a potential therapeutic alternative to sorafenib for the treatment of HCC.

MAPKs are key signaling proteins that regulate normal cell proliferation, survival and differentiation (30). Dysregulation of MAPK cascades plays an important role in HCC occurrence and development (31). The MAPK pathway comprises a cascade of phosphorylation events initiated from activated RAF proteins to MEK and MEK to ERK (32). RAF activity is strongly associated with cancer, and a diverse series of ATP-competitive RAF inhibitors has been developed over the past decade (33). Some of these first-generation RAF inhibitors, such as the BRAF inhibitors vemurafenib and dabrafenib, have markedly inhibited BRAFV600E-dependent melanomas due to inhibition of the monomeric form of this specific BRAF-mutant protein (34-36). However, in wild-type BRAF tumors bearing activating RAS mutations or increased RTK signaling, BRAF inhibitors were found to paradoxically induce RAF activation by RAF dimerization, leading to downstream ERK signaling and therefore enhancing tumor cell proliferation (7,37-39). To circumvent the limitation of first-generation
RAF inhibitors, a broad set of pan-RAF inhibitors were identified, including sorafenib, LY3009120, TAK632, CCT196969 and CCT241161 (40-43). Pan-RAF inhibitors such as sorafenib can inhibit BRAF or CRAF with high affinity (44). Though pan-RAF inhibitors induce BRAF-CRAF dimerization, they ultimately inhibit the phosphorylation of downstream MEK and ERK, confirming their efficacy in inhibiting the kinase activity of BRAF-CRAF heterodimers (41). PLC/PRF/5 cells contain an activating mutation in the KRAS gene that may drive their aberrant proliferation with a greater dependence on signaling through the MAPK pathway for survival (4). The data in the present study demonstrate that the response of the MAPK cascade to CBI-5725 resembles its response to sorafenib, suggesting that CBI-5725 may also be a pan-RAF inhibitor. CBI-5725 can block MEK/ERK signaling in PLC/PRF/5 cells harboring mutant K-RAS and wild-type BRAF.

Furthermore, cell cycle analysis by flow cytometry revealed a significantly greater accumulation of cells in $\mathrm{G} 2 / \mathrm{M}$ phase after treatment with $15 \mu \mathrm{M}$ CBI-5725 than after control treatment, indicating that CBI-5725 induces cell cycle arrest in $\mathrm{G} 2 / \mathrm{M}$ phase. Since only approximately $35.77 \%$ of tumor cells accumulated in G2/M phase, CBI-5725 did not elicit an irreversible cell cycle arrest but rather slowed the progression through this phase of the cell cycle, which contributed to the inhibition of cell proliferation (45). By contrast, the cell cycle distribution was nearly unaffected by $15 \mu \mathrm{M}$ sorafenib, which complied with the result of a previous study (3) that the cell cycle distribution of PLC/PRF/5 cells was not affected by $24 \mathrm{~h}$ of treatment with sorafenib at 3,10 , or $15 \mu \mathrm{M} . \mathrm{G} 2 / \mathrm{M}$ phase arrest relates to the down-regulation of cdc2, cdc $25 \mathrm{c}$ and cyclin B levels, as well as the up-regulation of p21 and p-cdc2 levels (46). Consequently, these regulators may be associated with the inhibition of the G2/M transition by CBI-5725.

Apoptosis, a tightly programmed cell death process, plays a critical role not only in the growth and homeostasis of normal tissues but also in the treatment of cancer, as it is a target of many therapeutic approaches. In this study, the CBI-5725-induced apoptosis of PLC/PRF/5 cells was examined by an Annexin V-PI assay, and a remarkable dose-dependent effect was detected. Moreover, CBI-5725 was shown to induce apoptosis more potently than sorafenib. The mechanism of the action of CBI-5725 on the apoptotic pathway was explored. Caspases are fundamental in apoptosis pathways because they are both initiators and executioners. Caspases can be activated through two common pathways. The intrinsic and extrinsic pathways converge on caspase-3, a downstream executioner caspase. Once activated, pro-caspase- 3 is cleaved to produce the active form of caspase-3, which is primarily responsible for PARP cleavage during cell death (47-49). PARP is an active participant in pivotal biological processes such as transcription and cell cycle modulation, the DNA damage response, apoptosis and genome integrity maintenance (50). Cleavage of PARP promotes cell destruction and functions as a marker of apoptotic cells (51). After treatment with CBI-5725, the levels of pro-caspase-3 and PARP decreased, while cleaved PARP levels increased, indicating that apoptosis induced by $\mathrm{CBI}-5725$ in $\mathrm{PLC} / \mathrm{PRF} / 5$ cells proceeded through the 
caspase-dependent pathway. However, sorafenib did not remarkably alter pro-caspase-3, PARP and cleaved PARP levels, suggesting that sorafenib-induced apoptosis might not rely on caspase activation in PLC/PRF/5 cells. This result is consistent with previous findings (3).

In the PLC/PRF/5 tumor xenograft experiments, CBI-5725 robustly prevented tumor growth. CBI-5725 at doses from 6 to $18 \mathrm{mg} / \mathrm{kg}$ produced nearly complete tumor growth inhibition after 14 days of oral administration. On the other hand, complete tumor growth inhibition was not achieved with sorafenib tosylate at doses up to $20 \mathrm{mg} / \mathrm{kg}$. The above results obtained from the in vitro experiments imply that blockage of the RAF/MEK/ERK pathway, induction of cell cycle arrest and initiation of apoptosis may lead to the tumor growth inhibition observed in PLC/PRF/5 tumor xenografts treated with CBI-5725.

The findings described herein are promising and should be verified in other HCC cell lines, such as HepG2 cells. The mechanisms underlying the superior antitumor efficacy of CBI-5725 remain to be determined.

In conclusion, this study demonstrates that CBI-5725 strongly inhibits HCC cell proliferation in vitro by blocking the RAF/MEK/ERK pathway to the same extent as sorafenib, elicits $\mathrm{G} 2 / \mathrm{M}$ cell cycle arrest and induces tumor cell apoptosis more potently than sorafenib. These findings may contribute to the remarkable antitumor efficacy of CBI-5725 against a human HCC xenograft model. Therefore, CBI-5725 may be an alternative to sorafenib for the treatment of liver cancer patients.

\section{Acknowledgements}

We greatly appreciate the technical support and discussions provided by previous members of the lab.

\section{Funding}

The present study was supported by the NSFC (grant no. 81503158) and The Beijing Municipal Administration of Hospitals' Youth Program (grant no. QML20160808).

\section{Availability of data and materials}

The datasets used and analyzed during the present study are available from the corresponding author upon reasonable request.

\section{Authors' contributions}

WW conceived, planned and carried out the experiments, and analyzed and interpreted the data. BX performed the flow cytometry to examine the cell cycle distribution and apoptosis. WW wrote the manuscript with support from QXL, DCJ and SYY. QXL contributed to study design, DCJ assisted with data interpretation, and SYY provided critical revision and helped shape the research, analysis and manuscript. All authors read and approved the final manuscript.

\section{Ethics approval and consent to participate}

Experiments using mice were performed in accordance with protocols approved by the Animal Ethical Committee of Xuanwu Hospital (Beijing, China).

\section{Consent for publication}

Not applicable.

\section{Competing interests}

The present study included the use of the compound CBI-5725; the patent for which is owned by Crown Bioscience, Inc. (Beijing, China). The author, Dr Qixiang Li, is affiliated with this company.

\section{References}

1. Lamarca A, Mendiola M and Barriuso J: Hepatocellular carcinoma: Exploring the impact of ethnicity on molecular biology. Crit Rev Oncol Hematol 105: 65-72, 2016.

2. Swamy SG, Kameshwar VH, Shubha PB, Looi CY, Shanmugam MK, Arfuso F, Dharmarajan A, Sethi G, Shivananju NS and Bishayee A: Targeting multiple oncogenic pathways for the treatment of hepatocellular carcinoma. Target Oncol 12: 1-10, 2016.

3. Liu L, Cao Y, Chen C, Zhang X, McNabola A, Wilkie D, Wilhelm S, Lynch $M$ and Carter C: Sorafenib blocks the $\mathrm{RAF} / \mathrm{MEK} / \mathrm{ERK}$ pathway, inhibits tumor angiogenesis and induces tumor cell apoptosis in hepatocellular carcinoma model PLC/PRF/5. Cancer Res 66: 11851-11858, 2006.

4. Wilhelm SM, Carter C, Tang L, Wilkie D, McNabola A, Rong H, Chen C, Zhang X, Vincent P, McHugh M, et al: BAY 43-9006 exhibits broad spectrum oral antitumor activity and targets the RAF/MEK/ERK pathway and receptor tyrosine kinases involved in tumor progression and angiogenesis. Cancer Res 64: 7099-7109, 2004

5. Azumi J, Tsubota T, Sakabe T and Shiota G: miR-181a induces sorafenib resistance of hepatocellular carinoma cells through downregulation of RASSF1 expression. Cancer Sci 107: 1256-1262, 2016.

6. Ezzoukhry Z, Louandre C, Trécherel E, Godin C, Chauffert B, Dupont S, Diouf M, Barbare JC, Mazière JC and Galmiche A: EGFR activation is a potential determinant of primary resistance of hepatocellular carcinoma cells to sorafenib. Int J Cancer 131: 2961-2969, 2012

7. Poulikakos PI, Zhang C, Bollag G, Shokat KM and Rosen N: RAF inhibitors transactivate RAF dimers and ERK signalling in cells with wild-type BRAF. Nature 464: 427-430, 2010.

8. Lackner MR, Wilson TR and Settleman J: Mechanisms of acquired resistance to targeted cancer therapies. Future Oncol 8: 999-1014, 2012.

9. Bagrodia S, Smeal T and Abraham RT: Mechanisms of intrinsic and acquired resistance to kinase-targeted therapies. Pigment Cell Melanoma Res 25: 819-831, 2012.

10. Bottsford-Miller JN, Coleman RL and Sood AK: Resistance and escape from antiangiogenesis therapy: Clinical implications and future strategies. J Clin Oncol 30: 4026-4034, 2012.

11. Moriguchi M, Umemura A and Itoh Y: Current status and future prospects of chemotherapy for advanced hepatocellular carcinoma. Clin J Gastroenterol 9: 184-190, 2016.

12. Pillet $\mathrm{S}$ and von Messling V: Canine distemper virus selectively inhibits apoptosis progression in infected immune cells. J Virol 83: 6279-6287, 2009.

13. Thomas E, Gopalakrishnan V, Somasagara RR, Choudhary B and Raghavan SC: Extract of Vernonia condensata, inhibits tumor progression and improves survival of tumor-allograft bearing mouse. Sci Rep 6: 23255, 2016.

14. Brauchle E, Thude S, Brucker SY and Schenke-Layland K: Cell death stages in single apoptotic and necrotic cells monitored by Raman microspectroscopy. Sci Rep 4: 4698, 2014.

15. Wlodkowic D, Telford W, Skommer J and Darzynkiewicz Z: Apoptosis and beyond: Cytometry in studies of programmed cell death. Methods Cell Biol 103: 55-98, 2011.

16. Kuo WT, Ho YJ, Kuo SM, Lin FH, Tsai FJ, Chen YS, Dong GC and Yao CH: Induction of the mitochondria apoptosis pathway by phytohemagglutinin erythroagglutinating in human lung cancer cells. Ann Surg Oncol 18: 848-856, 2011. 
17. Lam CR, Tan MJ, Tan SH, Tang MB, Cheung PC and Tan NS: TAK1 regulates SCF expression to modulate PKB $\alpha$ activity that protects keratinocytes from ROS-induced apoptosis. Cell Death Differ 18: 1120-1129, 2011.

18. Yang LJ, Chen Y, He J, Yi S, Wen L, Zhao J, Zhang BP and Cui GH: Betulinic acid inhibits autophagic flux and induces apoptosis in human multiple myeloma cells in vitro: Acta Pharmacol Sin 33: 1542-1548, 2012.

19. Buscà R, Pouysségur J and Lenormand P: ERK1 and ERK2 map kinases: Specific roles or functional redundancy. Front Cell Dev Biol 4: 53, 2016.

20. Robinson MJ and Cobb MH: Mitogen-activated protein kinase pathways. Curr Opin Cell Biol 9: 180-186, 1997.

21. Hoshino R, Chatani Y, Yamori T, Tsuruo T, Oka H, Yoshida O, Shimada Y, Ari-i S, Wada H, Fujimoto J and Kohno M: Constitutive activation of the $41-/ 43-\mathrm{kDa}$ mitogen-activated protein kinase signaling pathway in human tumors. Oncogene 18: 813-822, 1999

22. Zhang Q, Wei L, Yang H, Yang W, Yang Q, Zhang Z, Wu K and $\mathrm{Wu} \mathrm{J}$ : Bromodomain containing protein represses the Ras/Raf/MEK/ERK pathway to attenuate human hepatoma cell proliferation during HCV infection. Cancer Lett 371: 107-116, 2016.

23. Pucci B, Kasten M and Giordano A: Cell cycle and apoptosis. Neoplasia 2: 291-299, 2000.

24. Evan GI and Vousden KH: Proliferation, cell cycle and apoptosis in cancer. Nature 411: 342-348, 2001.

25. Wu H, Che X, Zheng Q, Wu A, Pan K, Shao A, Wu Q, Zhang J and Hong Y: Caspases: A molecular switch node in the crosstalk between autophagy and apoptosis. Int J Biol Sci 10: 1072-1083, 2014

26. Wong RS: Apoptosis in cancer: From pathogenesis to treatment. J Exp Clin Cancer Res 30: 87, 2011.

27. Fulda $\mathrm{S}$ and Debatin KM: Extrinsic versus intrinsic apoptosis pathways in anticancer chemotherapy. Oncogene 25: 4798-4811, 2006.

28. Duriez PJ and Shah GM: Cleavage of poly(ADP-ribose) polymerase: A sensitive parameter to study cell death. Biochem Cell Biol 75: 337-349, 1997.

29. Prieto-Domínguez N, Ordóñez R, Fernández A, García-Palomo A, Muntané J, González-Gallego J and Mauriz JL: Modulation of autophagy by sorafenib: Effects on treatment response. Front Pharmacol 7: 151, 2016.

30. Roberts PJ and Der CJ: Targeting the Raf-MEK-ERK mitogen-activated protein kinase cascade for the treatment of cancer. Oncogene 26: 3291-3310, 2007.

31. Li L, Zhao GD, Shi Z, Qi LL, Zhou LY and Fu ZX: The Ras/Raf/MEK/ERK signaling pathway and its role in the occurrence and development of HCC. Oncol Lett 12: 3045-3050, 2016

32. Jin T, Lavoie H, Sahmi M, David M, Hilt C, Hammell A and Therrien M: RAF inhibitors promote RAS-RAF interaction by allosterically disrupting RAF autoinhibition. Nat Commun 8: 1211,2017

33. Holderfield M, Deuker MM, McCormick F and McMahon M: Targeting RAF kinases for cancer therapy: BRAF-mutated melanoma and beyond. Nat Rev Cancer 14: 455-467, 2014

34. Chapman PB, Hauschild A, Robert C, Haanen JB, Ascierto P, Larkin J, Dummer R, Garbe C, Testori A, Maio M, et al: Improved survival with vemurafenib in melanoma with BRAF V600E mutation. N Engl J Med 364: 2507-2516, 2011.

35. Hauschild A, Grob JJ, Demidov LV, Jouary T, Gutzmer R, Millward M, Rutkowski P, Blank CU, Miller WH Jr, Kaempgen E, et al: Dabrafenib in BRAF-mutated metastatic melanoma: A multicentre, open-label, phase 3 randomised controlled trial. Lancet 380: 358-365, 2012.

36. Yao Z, Torres NM, Tao A, Gao Y, Luo L, Li Q, de Stanchina E, Abdel-Wahab O, Solit DB, Poulikakos PI and Rosen N: BRAF mutants evade ERK-dependent feedback by different mechanisms that determine their sensitivity to pharmacologic inhibition. Cancer Cell 28: 370-383, 2015.
37. Hatzivassiliou G, Song K, Yen I, Brandhuber BJ, Anderson DJ, Alvarado R, Ludlam MJ, Stokoe D, Gloor SL, Vigers G, et al: RAF inhibitors prime wild-type RAF to activate the MAPK pathway and enhance growth. Nature 464: 431-435, 2010.

38. Heidorn SJ, Milagre C, Whittaker S, Nourry A, Niculescu-Duvas I, Dhomen N, Hussain J, Reis-Filho JS, Springer CJ, Pritchard C and Marais R: Kinase-dead BRAF and oncogenic RAS cooperate to drive tumor progression through CRAF. Cell 140: 209-221, 2010.

39. Prahallad A, Sun C, Huang S, Di Nicolantonio F, Salazar R, Zecchin D, Beijersbergen RL, Bardelli A and Bernards R: Unresponsiveness of colon cancer to BRAF(V600E) inhibition through feedback activation of EGFR. Nature 483: 100-103, 2012.

40. Cox AD and Der CJ: The raf inhibitor paradox: Unexpected consequences of targeted drugs. Cancer Cell 17: 221-223, 2010.

41. Peng SB, Henry JR, Kaufman MD, Lu WP, Smith BD, Vogeti S, Rutkoski TJ, Wise S, Chun L, Zhang Y, et al: Inhibition of RAF isoforms and active dimers by LY3009120 leads to anti-tumor activities in RAS or BRAF mutant cancers. Cancer Cell 28: 384-398, 2015

42. Nakamura A, Arita T, Tsuchiya S, Donelan J, Chouitar J, Carideo E, Galvin K, Okaniwa M, Ishikawa T and Yoshida S: Antitumor activity of the selective pan-RAF inhibitor TAK-632 in BRAF inhibitor-resistant melanoma. Cancer Res 73: 7043-7055, 2013.

43. Girotti MR, Lopes F, Preece N, Niculescu-Duvaz D, Zambon A, Davies L, Whittaker S, Saturno G, Viros A, Pedersen M, et al: Paradox-breaking RAF inhibitors that also target SRC are effective in drug-resistant braf mutant melanoma. Cancer Cell 31: 466, 2017.

44. Knievel J, Schulz WA, Greife A, Hader C, Lübke T, Schmitz I, Albers P and Niegisch G: Multiple mechanisms mediate resistance to sorafenib in urothelial cancer. Int J Mol Sci 15: 20500-20517, 2014.

45. Weber GF, Gaertner FC, Erl W, Janssen KP, Blechert B, Holzmann B, Weighardt $\mathrm{H}$ and Essler M: IL-22-mediated tumor growth reduction correlates with inhibition of ERK1/2 and AKT phosphorylation and induction of cell cycle arrest in the G2-M phase. J Immunol 177: 8266-8272, 2006.

46. Cheng Y, Qiu F, Ye YC, Tashiro S, Onodera S and Ikejima T: Oridonin induces $\mathrm{G} 2 / \mathrm{M}$ arrest and apoptosis via activating ERK-p53 apoptotic pathway and inhibiting PTK-Ras-Raf-JNK survival pathway in murine fibrosarcoma L929 cells. Arch Biochem Biophys 490: 70-75, 2009.

47. Nicholson DW, Ali A, Thornberry NA, Vaillancourt JP, Ding CK, Gallant M, Gareau Y, Griffin PR, Labelle M, Lazebnik YA, et al: Identification and inhibition of the ICE/CED-3 protease necessary for mammalian apoptosis. Nature 376: 37-43, 1995.

48. Tewari M, Quan LT, O'Rourke K, Desnoyers S, Zeng Z, Beidler DR, Poirier GG, Salvesen GS and Dixit VM: Yama/CPP32 beta, a mammalian homolog of CED-3, is a CrmA-inhibitable protease that cleaves the death substrate poly(ADP-ribose) polymerase. Cell 81: 801-809, 1995.

49. Le RY, Kirkland JB and Shah GM: Cellular responses to DNA damage in the absence of Poly(ADP-ribose) polymerase. Biochem Biophys Res Commun 245: 1-10, 1998.

50. Vizetto-Duarte C, Custodio L, Gangadhar KN, Lago JH, Dias C, Matos AM, Neng N, Nogueira JM, Barreira L, Albericio F, et al: Isololiolide, a carotenoid metabolite isolated from the brown alga Cystoseira tamariscifolia, is cytotoxic and able to induce apoptosis in hepatocarcinoma cells through caspase-3 activation, decreased $\mathrm{Bcl}-2$ levels, increased $\mathrm{p} 53$ expression and PARP cleavage. Phytomedicine 23: 550-557, 2016.

51. Oliver FJ, de la Rubia G, Rolli V, Ruiz-Ruiz MC, de Murcia G and Murcia JM: Importance of poly(ADP-ribose) polymerase and its cleavage in apoptosis. Lesson from an uncleavable mutant. J Biol Chem 273: 33533-33955, 1998 